\title{
Molecular dynamics of SWNT/epoxy nanocomposites
}

\author{
Nicholas A. Fasanella* and Veera Sundararaghavan ${ }^{\dagger}$ \\ University of Michigan, Ann Arbor, MI, 48105, USA
}

\begin{abstract}
Molecular dynamics simulations are performed to compute thermal and mechanical properties of cured epoxy resins reinforced with pristine and covalently functionalized carbon nanotubes. A DGEBA-DDS epoxy network was built using the 'dendrimer' growth approach where $75 \%$ of available epoxy sites were cross-linked. The epoxy model is verified through comparisons to experiments, and simulations are performed on nanotube reinforced cross-linked epoxy matrix using the CVFF force field in LAMMPS. Full elastic stiffness matrices and linear coefficient of thermal expansion vectors are obtained for the nanocomposites. Large increases in stiffness and large decreases in thermal expansion were seen along the direction of the nanotube for both nanocomposite systems when compared to neat epoxy. The direction transverse to nanotube saw a $40 \%$ increase in stiffness due to covalent functionalization over neat epoxy at $1 \mathrm{~K}$ whereas the pristine nanotube system only saw a $7 \%$ increase due to van der Waal effects. The functionalized SWNT/epoxy nanocomposite showed an additional $42 \%$ decrease in thermal expansion along the nanotube direction when compared to the pristine SWNT/epoxy nanocomposite.
\end{abstract}

\section{Nomenclature}

E $\quad$ Elastic modulus, $\mathrm{Pa}$

$G \quad$ Shear modulus, $\mathrm{Pa}$

$\nu \quad$ Poisson's ratio

$T$ Temperature, $\mathrm{K}$ or ${ }^{\circ} \mathrm{C}$

$P \quad$ Pressure, atm

$L \quad$ Length, $\mathrm{m}$

$t \quad$ Time, s

$\alpha \quad$ Linear coefficient of thermal expansion, $1 /{ }^{\circ} \mathrm{C}$

Subscript

i Variable number

\section{Introduction}

Carbon nanotubes (CNTs) have been researched extensively in the last 20 years due to their outstanding mechanical, ${ }^{1-3}$ electrical, ${ }^{4-6}$ and thermal properties. ${ }^{7-8}$ Due to these exceptional properties and very high aspect ratios, forming CNT-polymer nanocomposites has become an attractive option to improve the properties of the polymer. This work will focus in particular on Single Wall Carbon Nanotubes (SWNTs). SWNTs are an atom thick single layer of graphene with a cylindrical structure with an elastic modulus around 1 TPa. ${ }^{9}$ Atomistic simulation methods allow us to characterize the effects of nanoparticles in a composite system without running costly experiments. Molecular Dynamics (MD) allows for the effects of mechanical and thermal loading to be isolated and visualized in regions of interest where it may not be possible with experiments. In this work, MD is used in order to explore the effects on the mechanical and dilatometric properties by adding pristine and covalently functionalized SWNTs to cross-linked polymers.

\footnotetext{
*Graduate Research Assistant, Aerospace Engineering, nickfas@umich.edu, AIAA Student Member.
}

${ }^{\dagger}$ Associate Professor, Aerospace Engineering, veeras@umich.edu, AIAA Senior Member. 
There has been numerous experimental studies on the effects of adding SWNTs to epoxies. ${ }^{10-23}$ To take full advantage of the mechanical and thermal properties of the SWNTs, attempts have been made to functionalize the nanotubes to the epoxy. Functionalization allows for better dispersion and interfacial bonding of the SWNTs to the polymer matrix, and has shown to give significantly improved mechanical properties. ${ }^{12-18}$ Attempts to study SWNT-epoxy systems via molecular dynamics are limited. Liang et. al used MD to study the interactions between SWNT and Epon 862 (DGEBF) resin and EPI-CURE W curing agent (DETDA). ${ }^{24}$ Gou et. al. expanded this work by studying and comparing affinities between SWNT and DGEBA (diglycidylether of bisphenol A)/diethylenetriamine (DETA). ${ }^{25}$ Zhu et. al studied SWNT-EPON 862 composites, and found stress-strain relations in the direction parallel to the nanotube for continuous and discontinuous CNTs. ${ }^{26}$ Mohan et. al. predicted the effects of carbon vacancy defects on a SWNT and EPON 862 (DGEBF) cross-linked with Curing Agent W (DETDA) composite. ${ }^{27}$ Molecular scale simulations allow the effects of the nanotube on the composite system to be isolated in ways not possible with experiments. Epoxies have an amorphous structure and building complex cross-linked molecular configurations with accurate properties is challenging. ${ }^{36}$ Using an amorphous cross-linked structure that has been experimentally verified, ${ }^{28}$ the thermomechanical properties of SWNT/epoxy composites will be investigated.

This paper is organized as follows: Section II outlines the chemistry of the epoxy chosen in this study. Section III describes methods to build nanotube reinforced cross-linked polymer composites using molecular dynamics. Section IV verifies the epoxy model by comparing the elastic and dilatometric properties to experimental data for the chosen epoxy system. Section V shows the thermal and mechanical results of the pristine SWNT/Epoxy and covalently functionalized SWNT/Epoxy nanocomposites. Section VI is conclusions.

\section{Materials}

Di-Glycidyl Ether of Bisphenol A (DGEBA) is the epoxy chosen for this study. The curing agent used is diamino diphenyl sulfone (DDS). The epoxy molecules were cross-linked (cured) with 3-3' diamino diphenyl sulfone (DDS). The structure of the epoxy and the amine can be seen in Fig. 1a and Fig. 1b respectively. The epoxy monomer has two epoxide (oxirane ring) groups with a total functionality of two, each epoxide has a cross-linking functionality of one towards the amine curing agents. The DDS monomer has two amine groups and a total functionality of four, each amine group with a functionality of two towards epoxy molecules. A typical amine stoichiometric ratio for synthetic epoxy is approximately $2: 1$ or $33.3 \mathrm{~mol} \%$ amine. The polymer is formed by bonding of the epoxide group in DGEBA and the amine groups in the DDS. To form a cross-link, the primary amine group reacts with the epoxide group, creating a bond between the terminal carbon of the epoxide group and the nitrogen of DDS. The carbon-oxygen bond breaks between the terminal carbon and the oxygen from the epoxide, and forms and alchohol $(-\mathrm{OH})$ link. The cross-linked structure seen in Fig. 2a can undergo another reaction with a different epoxy molecule, forming the final cross-linked structure seen in Fig. 2b.

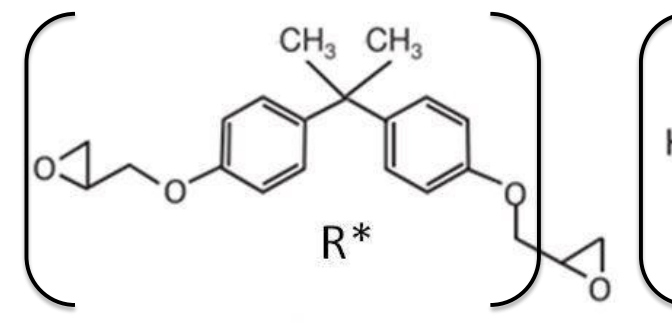

(a)

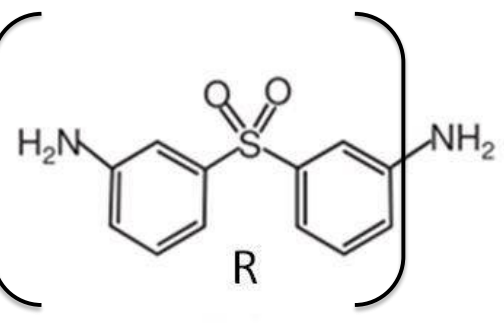

(b)

Figure 1. a. Chemical structure of epoxy resin, DGEBA and b. The amine monomer diamoino dipheyl sulfone. Brackets give the notation for $\mathbf{R}$ and $R^{*}$ in Fig. 2 


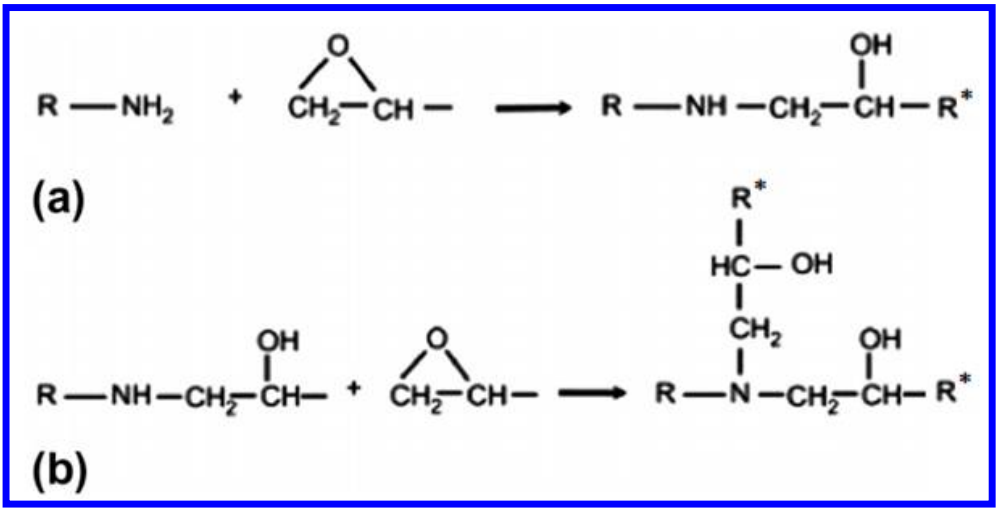

Figure 2. Epoxy-amine cross-linking through reaction of epoxide group and amine group

\section{Material Modeling}

For industrial grade epoxies, a conversion 70-95\% is typically seen when measured through Near-Infrared (NIR) Spectroscopy. ${ }^{29}$ The actual conversion percentage depends on processing conditions, namely the time and temperature of curing. In order to create a accurate models for molecular dynamics simulations, a number of approaches have been used to build cross-linked polymer networks that have greater than $70 \%$ cross link conversions. The majority of these approaches can be characterized as polymerizing unreacted monomer mixtures all-at-once (one-step) or over time (multi-step). For one-step cross-linking, sites are first randomly selected and then pairs of sites within a capture radius will be cross-linked together, ${ }^{30} .{ }^{31}$ The one-step methods lead to artificially high network strains. In multi-step cross linking, every reactive pair that satisfies a length criteria will be cross-linked. ${ }^{32-34}$ The length criteria increases with every iteration, and equilibration and cross-linking are repeated until desired cross-linking density is reached. Multi-step methods prevents and relieves network strains, but they are computationally expensive. In 2007, Christensen introduced a new method to build epoxy networks using a 'dendrimer' growth approach. ${ }^{35,36}$ In this approach, the thermoset resin is modeled by starting with a single monomer and then cross-linking a second layer of monomers around it. In the next step, a third layer of monomers are cross-linked to the second layer. In this way, generations (layers) of monomers are added to a seed structure that grows in size at every pass. The principal advantages of the dendrimer growth method are the complete avoidance of artificial network strain during curing and the low computational cost of the growing procedure. Fig. 3 shows a 3D dendrimer used to build the molecular model of a cross-linked epoxy structure. This approach is studied in this work for building realistic energy minimized polymer unit cells.

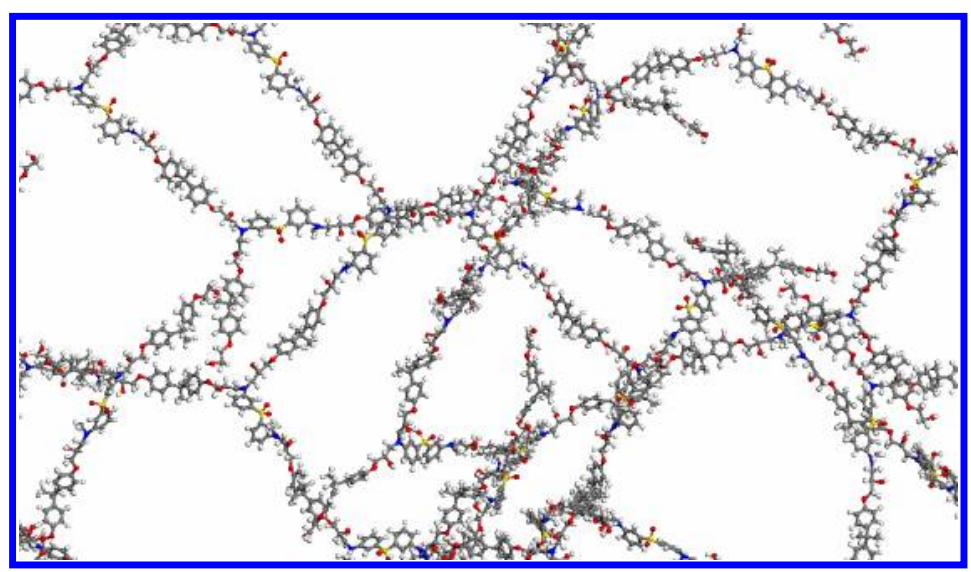

Figure 3. 3D dendrimer used to build the molecular model of a cross-linked epoxy structure.

The 'dendrimer' growth approach was used to build the epoxy network in Materials Studio ${ }^{41}$ containing 36 amine groups and 71 epoxy groups as seen in Fig. 4. The system contains 4601 atoms and is sufficiently 
complex to accurately capture the amorphous nature of the polymer. All simulations in this work are performed under periodic boundary conditions. $75 \%$ of available epoxy sites were cross-linked, which is representative of many structural epoxies. For all simulations in this study, the Consistent Valance Force Field $(\mathrm{CVFF})^{42}$ potential was used for bonded and non-bonded interactions in LAMMPS. ${ }^{43}$ This force-field has been used in a previous study to accurately predict thermodynamic properties of epoxy. ${ }^{44}$ To verify the accuracy of the initial dendrimer structure; the dilatometric curve simulated by MD will be compared to experimental results, and the full elastic stiffness matrix will be generated by conducting tensile and shear tests via molecular dynamics to verify the structure is isotropic.

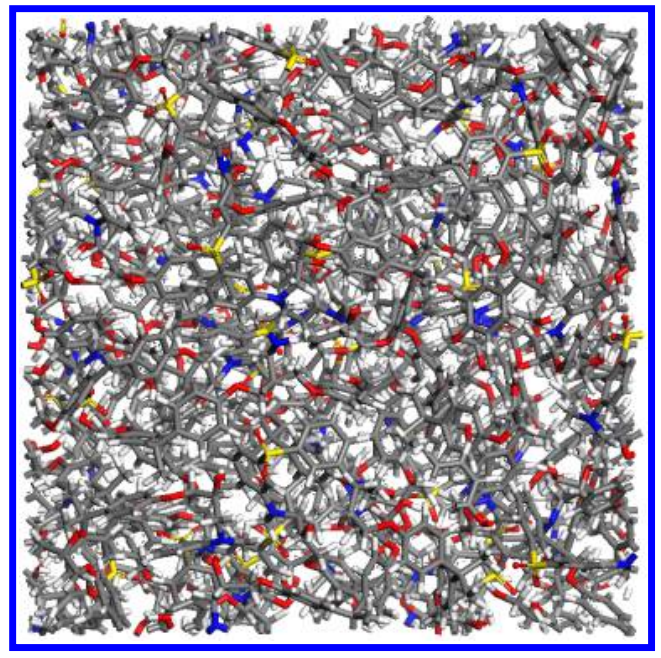

Figure 4. The dendrimer structure of epoxy after energy minimization.

To equilibriate the structure, the initial dendrimer is optimized by minimizing the energy using conjugate gradient (CG) minimize for 10000 iterations. Molecular dynamics was then performed over several annealing cycles using an NPT ensemble. Dynamics was first performed well above glass transition temperature (600 $\mathrm{K}$ and $1 \mathrm{~atm})$ for $500 \mathrm{ps}$. Next, dynamics was run well below $T_{g}(1 \mathrm{~K}$ and $1 \mathrm{~atm})$ for $500 \mathrm{ps}$, followed by $\mathrm{CG}$. This was done for ten cycles, when the potential energy was sufficiently minimized. A final density of $1.17 \mathrm{~g} / \mathrm{cc}$ at $1 \mathrm{~K}$ and $1 \mathrm{~atm}$ is obtained after convergence.

To build the CNT-Epoxy nanocomposites, a vacancy was created in the epoxy and an single walled armchair nanotube $(4,4)$ was inserted in the space. To equilibriate the structure, the same annealing process was run via a sequence of conjugate gradient (CG) minimization and dynamics above and below $T_{g}$. A functionalized SWNT/Epoxy composite was created by covalently bonding the nanotube to the DDS molecule. One of the two amine groups of the DDS is attached to the carbon nanotube, as shown in 5a. The other amine group reacts with the epoxide as shown earlier in Fig. 2. The degree of cross-linking is unaffected, as sites that were previously not reacted with the epoxy were selected to bond covalently to the nanotube. Experimentally, carbon nanotubes with amino groups covalently bonded to their side walls have been prepared by use of flourinated SWNTs. ${ }^{17,18,45,46}$ Again, equilibriation was performed via a sequence of conjugate gradient (CG) minimization and annealing until the structure was sufficiently minimized. The functionalized system containing four covalent bonds to the epoxy can be seen in Fig. 5b.

\section{Model Verification}

Tension and shear tests were run for neat epoxy model to obtain the full stiffness matrix. The epoxy is verified to be nearly isotropic by rotating the full orthotropic stiffness matrix in all directions as shown in Fig. 6a, and the isotropic properties of epoxy can be seen in Fig. 6b. It has been measured experimentally that for an $80 \%$ degree of cure for DGEBA-DDS resin system the tensile elastic modulus is $3.1 \mathrm{GPa}$ at room temperature $(300 \mathrm{~K}) .{ }^{48}$ Additionally, for tests in compression at room temperature and quasi-static loading rates, a Poisson's ratio of 0.37 has been measured experimentally. ${ }^{49}$ Despite the high strain rate of MD experiments, the Young's Modulus and Poisson's ratio at $300 \mathrm{~K}$ are found to be $2.9 \mathrm{GPa}$ and 0.35 respectively, which compares well with the quasi-static experimental results. 


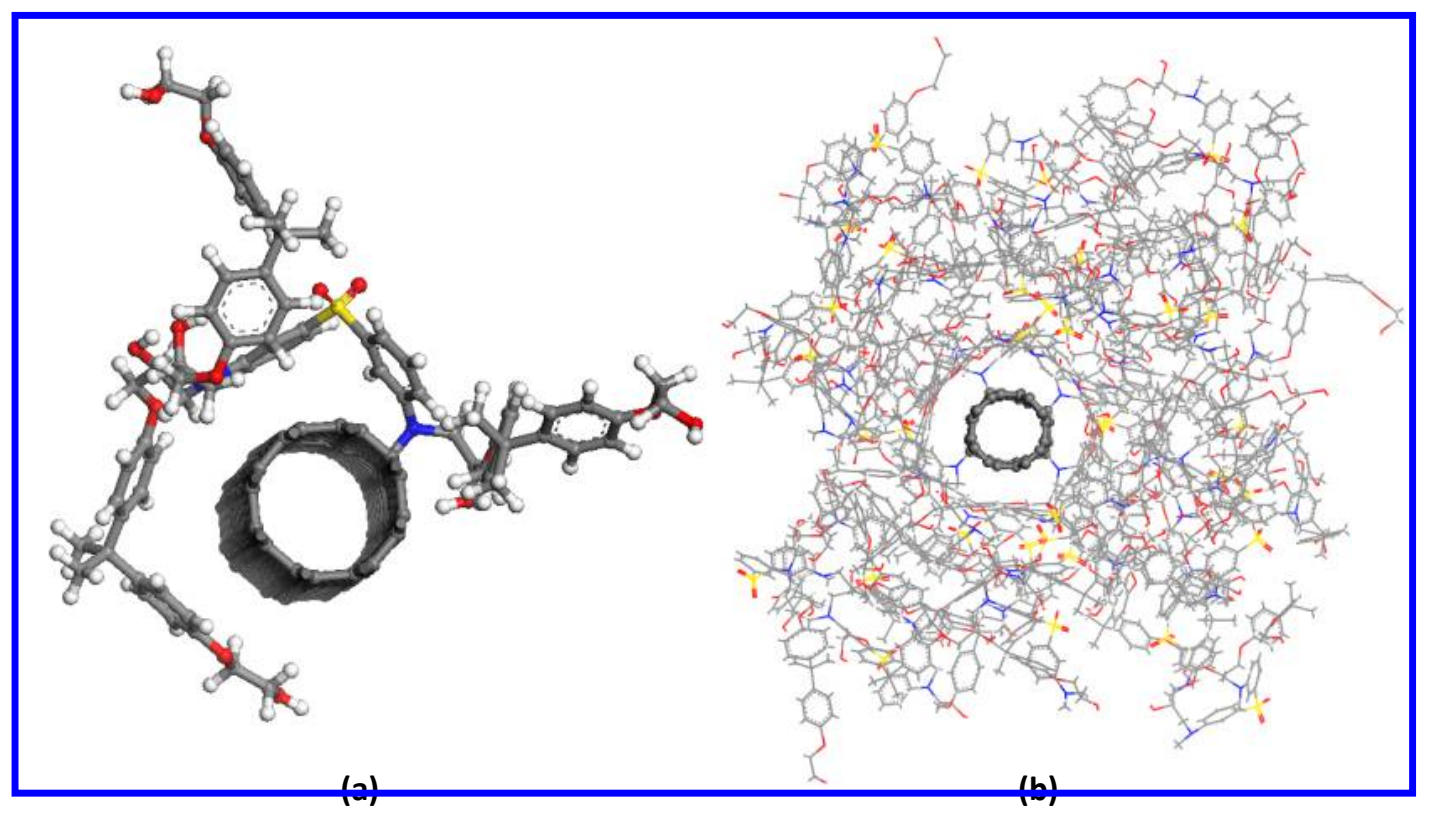

Figure 5. a. Nanotube functionalization to amine and subsequent cross-linking to epoxy. b. Functionalized Carbon nanotube and epoxy nanocomposite

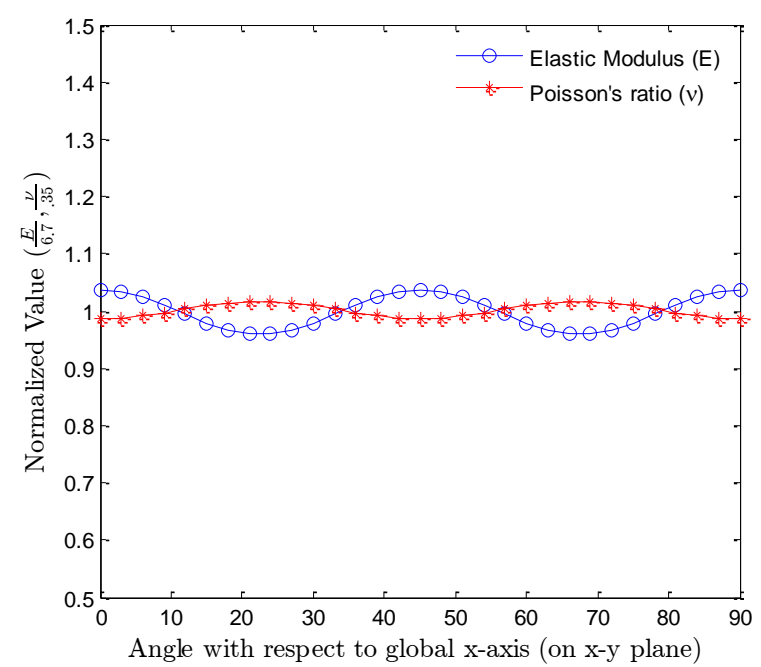

(a)

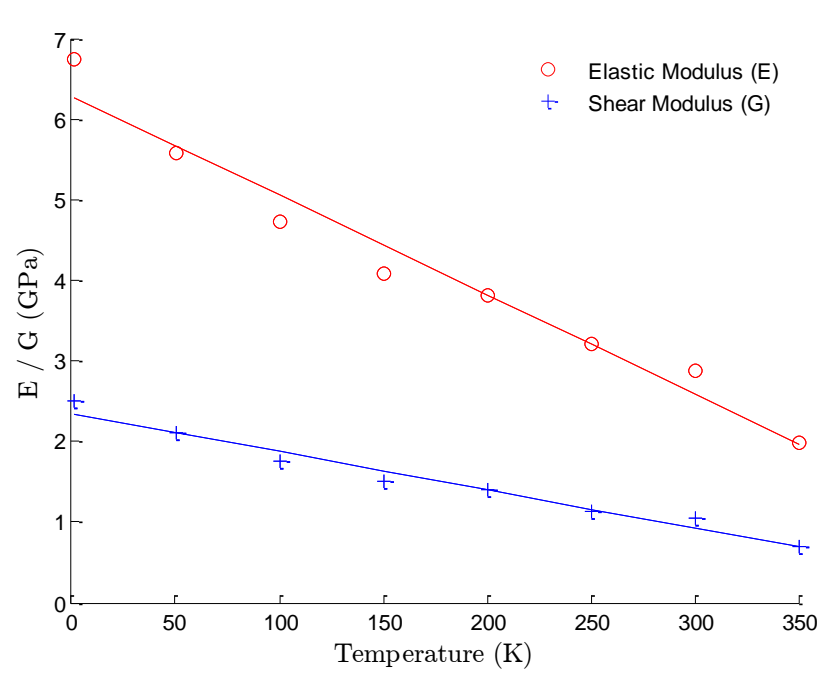

(b)

Figure 6. a. Variation of elastic properties along various directions in the $\mathrm{x}-\mathrm{y}$ plane. b. Elastic Modulus and Shear modulus for epoxy model.

The thermal expansion coefficient for the epoxy was obtained via an NPT simulation. The change in cell length with varying temperature is plotted with an experimentally measured dilatometric curve measured by Tcharkhtchi et. al. ${ }^{50}$ The experimental curve is in very good agreement with the MD calculated data in Fig. 7. The linear coefficient of thermal expansion between $223 \mathrm{~K}$ to $323 \mathrm{~K}$ is measured to be $54.6 \mu /{ }^{\circ} \mathrm{C}$ from this figure. 


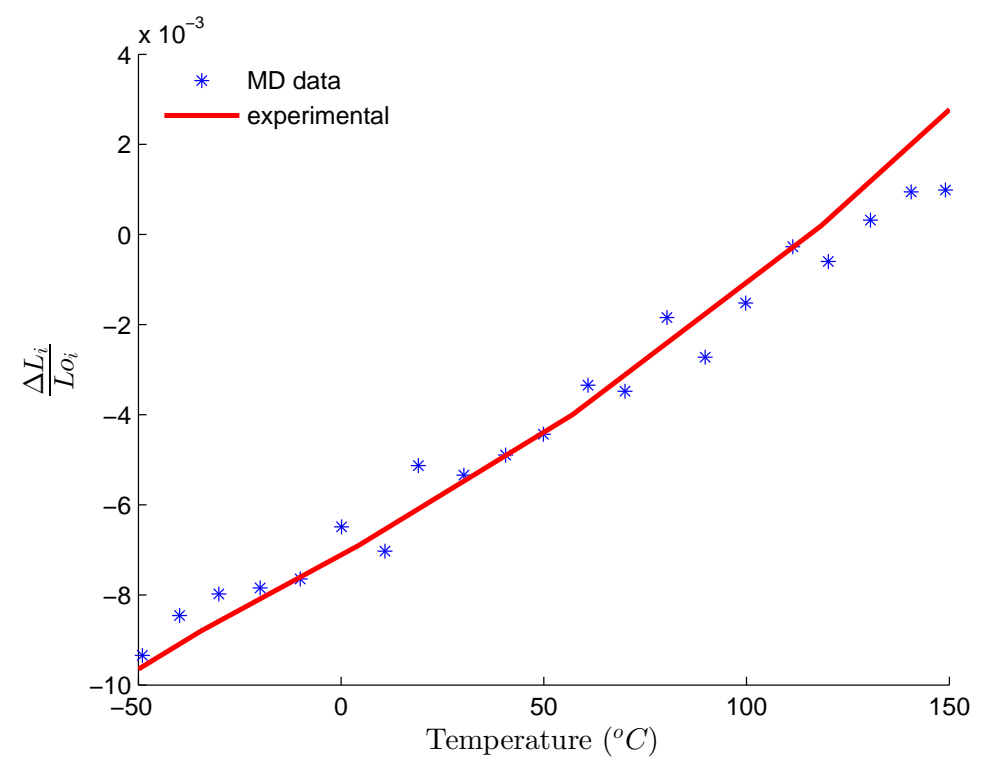

Figure 7. MD calculations of thermal expansion is superposed with experimentally measured dilatometric curves reported in Ref. ${ }^{50}$

\section{Results}

Similar to epoxy, tension and shear tests are run for the pristine and covalently functionalized SWNT/epoxy composite systems to obtain the full stiffness matrices. Both nanocomposite systems are found to be transversely isotropic and the properties of the pristine SWNT/epoxy system are seen in Table 1 and the covalently functionalized SWNT/epoxy system in Table 2. As expected, both systems see dramatically increased stiffness in the z-direction due to the inclusion of the nanotube. By comparing the properties of the covalently functionalized SWNT/epoxy composite to that of the pristine SWNT/epoxy composite, it is seen that the nanocomposite becomes much more stiff in the direction transverse to the nanotubes $\left(E_{x x}\right)$, as highlighted in Fig. 8. It can be readily seen here that the directions transverse to the nanotube see substantial improvement due to functionalization $(40 \%$ at $1 \mathrm{~K}$ ), where the pristine nanotube system only sees minor improvements in transverse properties due to van der Waal effects $(7 \%$ at $1 \mathrm{~K}) .{ }^{25}$ It is worth noting that functionalization causes $s p^{3}$ hybridization carbon sites on the sidewalls of the nanotube, and this lowers the stiffness of the composite system along the nanotube $\left(E_{z z}\right)$, as seen in Tables 1 and 2. It has been shown theoretically, however, that even a high degree of functionalization on the sidewall of the SWNT will only decrease the mechanical strength by $15 \% .{ }^{47}$ There are negligible differences in the in-plane Poisson's ratio $\left(v_{x y}\right)$ between the functionalized and non-functionalized systems. There is a clear reduction in the out of plane Poisson's ratio $\left(v_{z x}\right)$, meaning that there will be less contraction in the $\mathrm{x}-\mathrm{y}$ plane when the system is pulled along the nanotube due to the nanotube functionalization. Finally, it can be seen that the shear modulus in the $\mathrm{x}-\mathrm{y}$ plane has noticeably increased due to the covalent bonding to the SWNT.

The coefficient of thermal expansion is measured for the SWNT/epoxy composite with pristine nanotubes. The thermal expansion in the isotropic plane $(\mathrm{x}-\mathrm{y})$ and nanotube $(\mathrm{z})$ directions are shown in Fig. 9. Since the structure is transversely isotropic, the results in the $\mathrm{x}$ and $\mathrm{y}$ directions are identical and the linear coefficient of thermal expansion is measured to be $55.3 \mu /{ }^{\circ} \mathrm{C}$, which is roughly equal to the expansion of plain epoxy. This result is expected since there is no functionalizion to the nanotube, so the epoxy is free to expand in the $\mathrm{x}$-y plane. The expansion in the z-direction is found to be $6.9 \mu /{ }^{\circ} \mathrm{C}$, where the thermal expansion is constrained by the nanotube which has a longitudinal coefficient of thermal expansion of $-1.2 \mu /{ }^{\circ} \mathrm{C} .{ }^{52}$ For the covalently functionalized SWNT/epoxy composite, the thermal expansion in isotropic plane (x-y) and nanotube $(\mathrm{z})$ directions can be seen in Fig. 10. There is a negligible effect of the covalent bonding for thermal expansion on the $\mathrm{x}-\mathrm{y}$ direction. However, even with only 4 covalent bonds the thermal expansion is reduced to $4.3 \mu /{ }^{\circ} \mathrm{C}$ in the nanotube direction. This represents a $42 \%$ reduction when compared to the pristine SWNT/epoxy composite. 


\begin{tabular}{cccccc}
\hline Temperature $(\mathrm{K})$ & $E_{x x}(G P a)$ & $E_{z z}(G P a)$ & $\nu_{x y}$ & $\nu_{z x}$ & $G_{x y}(G P a)$ \\
\hline 1 & 7.2 & 72 & 0.49 & 0.32 & 2.4 \\
\hline 50 & 6.2 & 71 & 0.52 & 0.32 & 2.1 \\
\hline 100 & 5.9 & 70 & 0.54 & 0.36 & 2.0 \\
\hline 150 & 5.1 & 70 & 0.55 & 0.36 & 1.7 \\
\hline 200 & 4.7 & 69 & 0.59 & 0.37 & 1.5 \\
\hline 250 & 3.7 & 68 & 0.67 & 0.40 & 1.3 \\
\hline 300 & 2.7 & 68 & 0.75 & 0.43 & .87 \\
\hline 350 & 2.0 & 67 & 0.75 & 0.45 & .77 \\
\hline
\end{tabular}

Table 1. Material properties- Pristine SWNT/epoxy composite

\begin{tabular}{cccccc}
\hline Temperature $(\mathrm{K})$ & $E_{x x}(G P a)$ & $E_{z z}(G P a)$ & $\nu_{x y}$ & $\nu_{z x}$ & $G_{x y}(G P a)$ \\
\hline 1 & 9.4 & 70 & 0.49 & 0.30 & 3.3 \\
\hline 50 & 8.7 & 69 & 0.50 & 0.30 & 2.9 \\
\hline 100 & 8.0 & 69 & 0.54 & 0.31 & 2.8 \\
\hline 150 & 6.6 & 68 & 0.54 & 0.31 & 2.5 \\
\hline 200 & 6.0 & 67 & 0.57 & 0.32 & 2.2 \\
\hline 250 & 4.9 & 66 & 0.58 & 0.35 & 1.8 \\
\hline 300 & 4.0 & 66 & 0.63 & 0.38 & 1.6 \\
\hline 350 & 3.0 & 65 & 0.67 & 0.38 & .86 \\
\hline
\end{tabular}

Table 2. Material properties- Covalently functionalized SWNT/epoxy composite

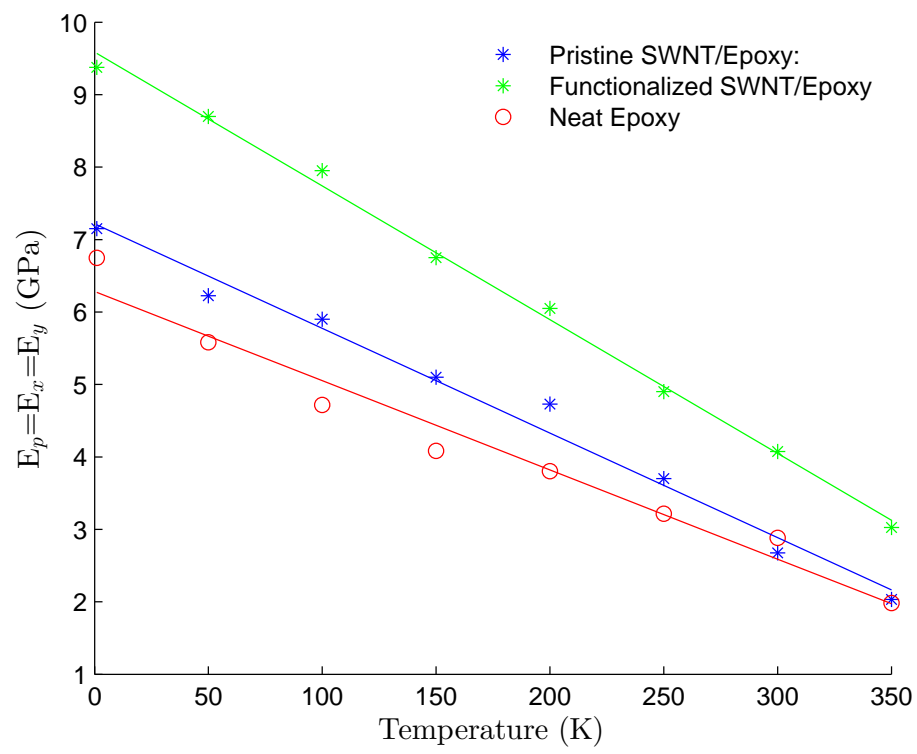

Figure 8. Elastic modulus in the plane of isotropy for the SWNT/epoxy composite systems and plain epoxy (fully isotropic) 


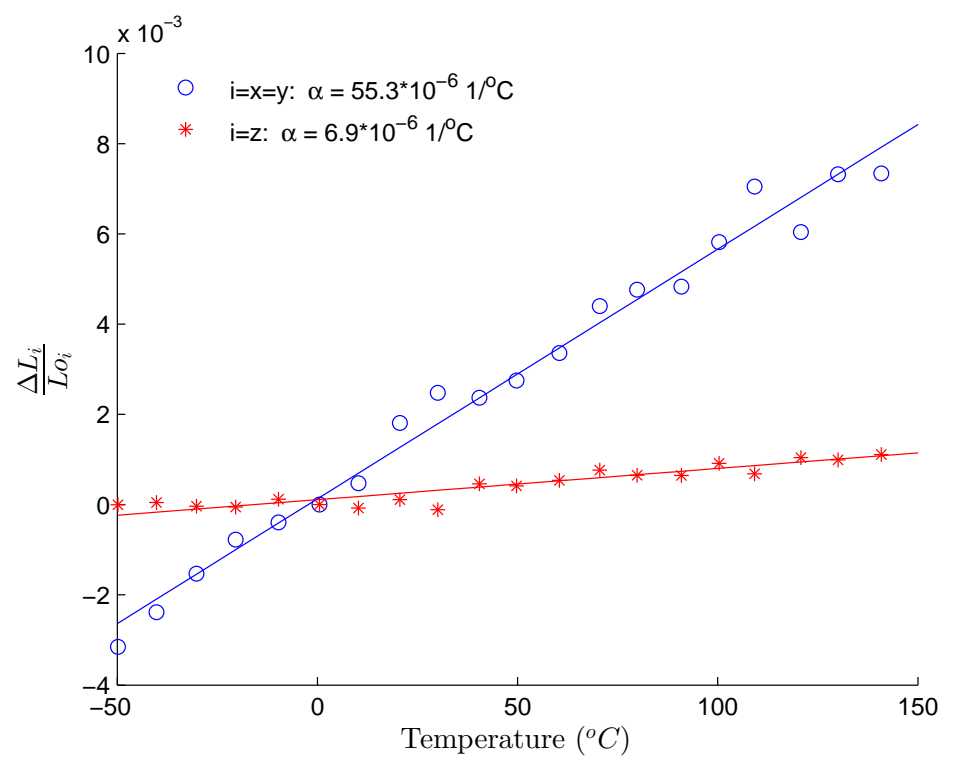

Figure 9. Thermal expansion for pristine SWNT/epoxy nanocomposite in the z-direction (along nanotube) and in plane of isotropy $(x-y)$.

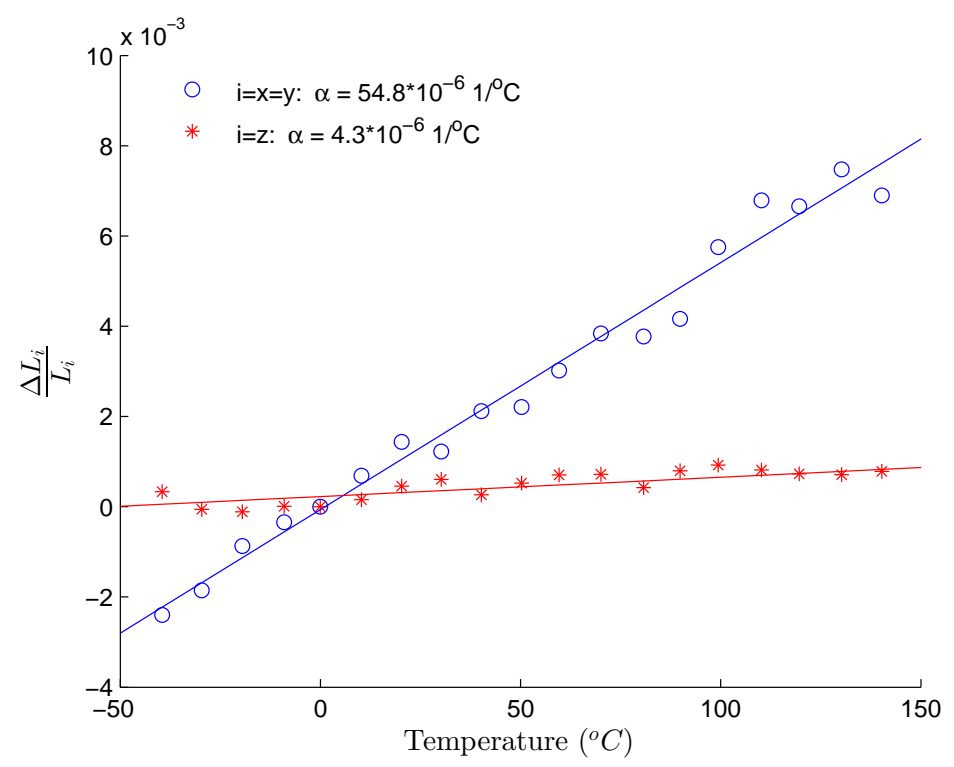

Figure 10. Thermal expansion for covalently functionalized SWNT/Epoxy nanocomposite in the z-direction (along nanotube) and in plane of isotropy (x-y).

We have explored the effects of randomly oriented nanotubes in order to simulate the effects of an isotropic system and to compare our results with experiments in a recent journal article. ${ }^{53}$ In order to do this, the stiffness matrices and thermal expansion vectors shown here are rotated and averaged over all possible orientations. It is found that adding randomly oriented nanotubes results in a huge gain in the elastic modulus for both the functionalized SWNT/epoxy composite (200\% increase at $1 \mathrm{~K})$ and pristine SWNT/epoxy composite ( $180 \%$ increase at $1 \mathrm{~K})$ over plain epoxy. The coefficient of thermal expansion is seen to be reduced by $30 \%$ for the pristine SWNT/epoxy composite system and 32\% for the covalently functionalized SWNT/epoxy composite system. ${ }^{53}$ It is shown that these thermal dilatation results agree well with experiments ${ }^{11,19}$ and that very high increases in stiffness can be achieved through wt\% of SWNTs. ${ }^{13,16,21}$ A summary of the dilatometric results for for the randomly oriented SWNT/epoxy composites can be seen in Fig. 11.

\section{8 of 11}




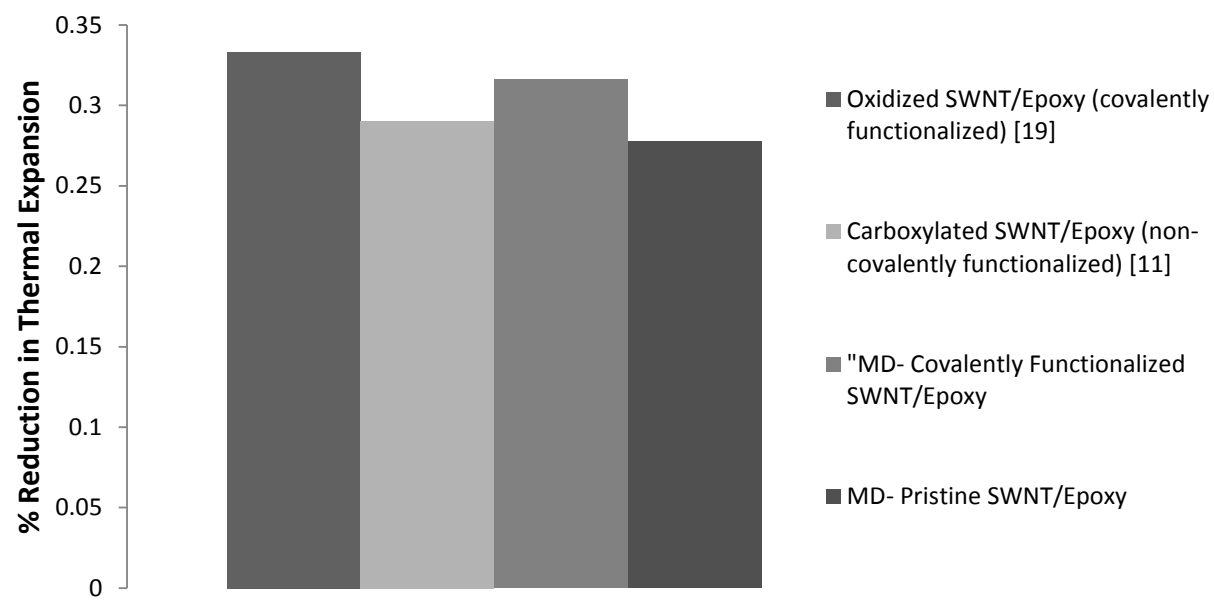

Figure 11. Experimental comparison in the percent reduction of the linear coefficient of thermal expansion due to the addition of SWNTs to epoxy ${ }^{53}$

\section{Conclusion}

Molecular dynamics has been used to analyze the thermal and mechanical properties of creating SWNT/ epoxy nanocomposites by adding functionalized and pristine carbon nanotubes to cross-linked polymers. A 4601 atom DGEBA-DDS epoxy network was built using the 'dendrimer' growth approach, where $75 \%$ of available epoxy sites were cross-linked. The epoxy model was verified by showing the system is isotropic, and by comparing the thermal expansion to experiments. The change in the mechanical and dilatometric properties was studied in every direction for both sets of SWNT/epoxy nanocomposites and the full stiffness matrices and thermal expansion vectors were obtained. As expected, there was huge increase in stiffness along the nanotube direction for both the pristine SWNT/epoxy and functionalized SWNT/epoxy composites. The direction transverse to the nanotube saw a $40 \%$ increase in stiffness due to covalent functionalization over neat epoxy at $1 \mathrm{~K}$, whereas the pristine nanotube system only saw a $7 \%$ increase due to van der Waal effects. The thermal expansion transverse to the nanotube was about equal to the neat epoxy for both nanocomposite systems. The thermal expansion along the nanotube was significantly decreased due to the negative coefficient of thermal expansion of a SWNT. An additional $42 \%$ reduction in thermal expansion in the direction of the nanotube was realized for the covanently functionalized SWNT/epoxy composite when compared to pristine SWNT/epoxy composite. Future work will consist of investigating other thermomechanical properties for the nanocomposite systems, such as thermal conductivity. Additionally, it is of interest to the authors to study the effects of curing conversion, as well as the effects of varying the chirality and number of walls of the nanotube.

\section{Acknowledgments}

The work presented here was supported by Boeing Company (Seattle, WA). The authors acknowledge the support of the University of Michigan Center for Advanced Computing (CAC), a high-performance computing center located in the Ann Arbor campus.

\section{References}

\footnotetext{
${ }^{1}$ Treacy, M.M., Ebbesen, T.W., Gibson, J.M., Exceptionally high Youngs modulus observed for individual carbon nanotubes, Nature, $381,678-680$.

${ }^{2}$ Yakobson, B., Mechanical properties of carbon nanotubes. Topics in Applied Physics, 80, 287-329.

${ }^{3}$ D.A. Walters, et al. Elastic strain of freely suspended single-wall carbon nanotube ropes, Applied Physics Letters, 74 (25), pp. 3803-3805.

${ }^{4}$ Thess, A., et al. Crystalline ropes of metallic carbon nanotubes, Science, 273, 483-487.

${ }^{5}$ Wildoer J.W.G., Venema L.C., Rinzler, A.G., Smalley, R.E., Dekker, C., Electronic structure of atomically resolved carbon nanotubes, Nature, 391, 5962.
} 
${ }^{6}$ Odom, T.W., Huang, J.L., Kim, P., Lieber, C.M., Atomic structure and electronic properties of single-walled carbon nanotubes, Nature, 391, pp. 6264.

${ }^{7}$ Dresselhaus, M.S.,P.C. Eklund. Photons in carbon nanotubes, Advances in Physics, 49(6), 705-814.

${ }^{8}$ Hone, J. "Phonons and Thermal Properties of Carbon Nanotubes", Topics in Applied Physics, 80, 273-287.

${ }^{9}$ Yu, M.F., Files, B.S., Arepalli, S., Ruoff, R.S., Tensile loading of ropes of single wall carbon nanotubes and their mechanical properties. Phys Rev Lett, 95, 5552-5555.

${ }^{10}$ Gojny, F.H., Wichmann, M.H.G., Fiedler, B., Kinloch, I.A., Bauhofer, W., Windle, A.H., Schulte, K., 2006. Evaluation and identification of electrical and thermal conduction mechanisms in carbon nanotube/epoxy composites. Polymer 47 , 20362045

${ }^{11}$ Pizzutto, C.E., Suave, J., Bertholdi, J., Pezzin, S.H., Coelho, L.A.F., Amico, S.C., 2010. Mechanical and Dilatometric Properties of Carboxylated SWCNT/Epoxy Composites: Effects of the Dispersion in the Resin and in the Hardener. Journal of Reinforced Plastics and Composites 29, 524530.

${ }^{12}$ Sun, L., Warren, G.L., OReilly, J.Y., Everett, W.N., Lee, S.M., Davis, D., Lagoudas, D., Sue, H.-J., 2008. Mechanical properties of surface-functionalized SWCNT/epoxy composites. Carbon 46, 320328.

${ }^{13}$ Zhu, J., Peng, H., Rodriguez-Macias, F., Margrave, J.L., Khabashesku, V.N., Imam, A.M., Lozano, K., Barrera, E.V., 2004. Reinforcing Epoxy Polymer Composites Through Covalent Integration of Functionalized Nanotubes. Advanced Functional Materials 14, 643648.

${ }^{14}$ Wang, S., Liang, Z., Liu, T., Wang, B., Zhang, C., 2006. Effective amino-functionalization of carbon nanotubes for reinforcing epoxy polymer composites. Nanotechnology 17, 15511557.

${ }^{15}$ Wang, S., Qiu, J., 2009. Modification of epoxy resins via $\mathrm{m}$-chloroperbenzoic acid-epoxidized carbon nanotubes. Journal of Applied Polymer Science 112, 33223326.

16 Wang, S., Liang, R., Wang, B., Zhang, C., 2008. Reinforcing polymer composites with epoxide-grafted carbon nanotubes. Nanotechnology 19, 085710.

${ }^{17}$ Zhu, J., Kim, J., Peng, H., Margrave, J.L., Khabashesku, V.N., Barrera, E.V., 2003. Improving the Dispersion and Integration of Single-Walled Carbon Nanotubes in Epoxy Composites through Functionalization. Nano Letters 3, 11071113.

${ }^{18}$ Valentini, L., Puglia, D., Carniato, F., Boccaleri, E., Marchese, L., Kenny, J.M., 2008. Use of plasma fluorinated singlewalled carbon nanotubes for the preparation of nanocomposites with epoxy matrix. Composites Science and Technology 68, 10081014.

${ }^{19}$ Wang, S., Liang, Z., Gonnet, P., Liao, Y.-H., Wang, B., Zhang, C., 2007. Effect of Nanotube Functionalization on the Coefficient of Thermal Expansion of Nanocomposites. Advanced Functional Materials 17, 8792.

${ }^{20}$ Wang, Q., Dai, J., Li, W., Wei, Z., Jiang, J., 2008. The effects of CNT alignment on electrical conductivity and mechanical properties of SWNT/epoxy nanocomposites. Composites Science and Technology 68, 16441648.

$\checkmark{ }^{21}$ Feng, Q.-P., Shen, X.-J., Yang, J.-P., Fu, S.-Y., Mai, Y.-W., Friedrich, K., 2011. Synthesis of epoxy composites with high carbon nanotube loading and effects of tubular and wavy morphology on composite strength and modulus. Polymer 52, 60376045 .

${ }^{22}$ Gong, X., Liu, J., Baskaran, S., Voise, R.D., Young, J.S., 2000. Surfactant-Assisted Processing of Carbon Nanotube/Polymer Composites. Chemistry of Materials 12, 10491052.

$\checkmark{ }^{23}$ De Villoria, R.G., Miravete, A., Cuartero, J., Chiminelli, A., Tolosana, N., 2006. Mechanical properties of SWNT/epoxy composites using two different curing cycles. Composites Part B: Engineering 37, 273277. doi:10.1016/j.compositesb.2006.01.002

$\checkmark{ }^{24}$ Liang, Z., Gou, J., Zhang, C., Wang, B., Kramer, L., 2004. Investigation of molecular interactions between (10, 10) single-walled nanotube and Epon 862 resin/DETDA curing agent molecules. Materials Science and Engineering: A 365, 228234.

$\checkmark{ }^{25}$ Gou, J., Fan, B., Song, G., Khan, A., 2006. Study of affinities between single-walled nanotube and epoxy resin using molecular dynamics simulation. International Journal of Nanoscience 5, 131144.

${ }^{26}$ Zhu, R., Pan, E., Roy, A.K., 2007. Molecular dynamics study of the stressstrain behavior of carbon-nanotube reinforced Epon 862 composites. Materials Science and Engineering: A 447, 5157.

${ }^{27}$ Mohan, R., Fefey, E., Kelkar, A., 2012. Predictive Mechanical Properties of EPON 862 (DGEBF) cross-linked with Curing Agent W (DETDA) and SWCNT using MD Simulations - Effect of Carbon Vacancy Defects. American Institute of Aeronautics and Astronautics.

${ }^{28}$ Sundararaghavan, V., Kumar, A., 2013. Molecular dynamics simulations of compressive yielding in cross-linked epoxies in the context of Argon theory. International Journal of Plasticity 47, 111125.

${ }^{29}$ Wang, Q., Storm, B.K., Houmller, L.P., 2003. Study of the isothermal curing of an epoxy prepreg by near-infrared spectroscopy. Journal of applied polymer science 87, 22952305.

${ }^{30}$ Yarovsky, I., Evans, E., 2002. Computer simulation of structure and properties of crosslinked polymers: application to epoxy resins. Polymer 43, 963969.

${ }^{31}$ Rottach, D.R., Curro, J.G., Budzien, J., Grest, G.S., Svaneborg, C., Everaers, R., 2007. Molecular Dynamics Simulations of Polymer Networks Undergoing Sequential Cross-Linking and Scission Reactions. Macromolecules 40, 131139.

${ }^{32}$ Heine, D.R., Grest, G.S., Lorenz, C.D., Tsige, M., Stevens, M.J., 2004. Atomistic Simulations of End-Linked Poly(dimethylsiloxane) Networks: Structure and Relaxation. Macromolecules 37, 38573864.

${ }^{33}$ Varshney, V., Patnaik, S.S., Roy, A.K., Farmer, B.L., 2008. A Molecular Dynamics Study of Epoxy-Based Networks: Cross-Linking Procedure and Prediction of Molecular and Material Properties. Macromolecules 41, 68376842.

${ }^{34} \mathrm{Wu}, \mathrm{C}$., Xu, W., 2006. Atomistic molecular modelling of crosslinked epoxy resin. Polymer 47, 60046009.

${ }^{35}$ Christensen, S., Atomistically Explicit Molecular Dynamics Simulations of Thermosetting Polymers. Proc. of 39th ISTC SAMPE Conf. 2007.

${ }^{36}$ Knox, C. K., J. W. Andzelm, J. L. Lenhart, A. R. Browning, and S. Christensen. High strain rate mechanical behavior of epoxy networks from molecular dynamics simulations. Proc. of 27th Army Science Conference. 2010.

${ }^{37}$ Kawamura, T., Kangawa, Y., Kakimoto, K., 2006. Investigation of thermal conductivity of nitride mixed crystals and superlattices by molecular dynamics. physica status solidi (c) 3, 16951699. 
${ }^{38}$ Chantrenne, P., Raynaud, M., Baillis, D., Barrat, J.L., Study of Phonon heat transfer in metallic solids from molecular dynamic simulations, Microscale Thermophys Eng, 7 117-136.

${ }^{39}$ Terao, T., Lussetti, E., Mller-Plathe, F., 2007. Nonequilibrium molecular dynamics methods for computing the thermal conductivity: Application to amorphous polymers. Physical Review E 75.

${ }^{40}$ Galamba, N., Nieto de Castro, C.A., Ely, J.F., 2004. Thermal conductivity of molten alkali halides from equilibrium molecular dynamics simulations. The Journal of Chemical Physics 120, 8676.

${ }^{41}$ Accelrys Software Inc., Discovery Studio Modeling Environment: Release 5.5, San Diego (2007).

${ }^{42}$ Dauber-Osguthorpe, P.V., Roberts, A, Osguthorpe, D.J., Wolff, J., Genest, M., Hagler, A.T., Structure and energetics of ligand binding to proteins: E. coli dihydrofolate reductase-trimethoprim, a drug-receptor system, Proteins: Structure, Function and Genetics, 4, 31-47.

${ }^{43}$ Plimpton, S., Fast Parallel Algorithms for Short-Range Molecular Dynamics, J Comp Phys, 117, 1-19.

${ }^{44}$ Varshney, V., Patnaik, S.S., Roy, A.K., Farmer, B.L., 2009. Heat transport in epoxy networks: A molecular dynamics study. Polymer 50, 33783385.

${ }^{45}$ Stevens, J.L., Huang, A.Y., Peng, H., Chiang, I.W., Khabashesku, V.N., Margrave, J.L., 2003. Sidewall AminoFunctionalization of Single-Walled Carbon Nanotubes through Fluorination and Subsequent Reactions with Terminal Diamines. Nano Letters 3, 331336.

${ }^{46}$ Zhao, Y., Barrera, E.V., 2010. Asymmmetric Diamino Functionalization of Nanotubes Assisted by BOC Protection and Their Epoxy Nanocomposites. Advanced Functional Materials 20, 30393044.

${ }^{47}$ Garg, A., Sinnott, S.B., 1998. Effect of chemical functionalization on the mechanical properties of carbon nanotubes. Chemical Physics Letters 295, 273278.

${ }^{48}$ White, S.R., Mather, P.T., Smith, M.J., 2002. Characterization of the cure-state of DGEBA-DDS epoxy using ultrasonic, dynamic mechanical, and thermal probes. Polymer Engineering \& Science 42, 5167.

${ }^{49}$ Heinz, S.R., Wiggins, J.S., 2010. Uniaxial compression analysis of glassy polymer networks using digital image correlation. Polym. Test. 29, 925932.

${ }^{50}$ Tcharkhtchi, A., Gouin, E., Verdu, J., 2000. Thermal expansion of epoxideamine networks in the glassy state. Journal of Polymer Science Part B: Polymer Physics 38, 537543.

${ }^{51}$ Christensen, S., 2009. Using molecular dynamics coupled with higher length scale simulations for the development of improved composite matrix materials. Proc. of 17th Int. Conf. on Composite Materials. 2009.

${ }^{52}$ Kwon, Y.-K., Berber, S., Tomnek, D., 2004. Thermal Contraction of Carbon Fullerenes and Nanotubes. Physical Review Letters 92 .

${ }^{53}$ Fasanella, N., Sundararaghavan, V., Atomistic modeling of thermomechanical properties of SWNT/Epoxy nanocomposites, Modelling and Simulation in Materials Science and Engineering, Submitted: MSMSE-100980, 2015. 